\title{
Contemporary rates and predictors of open conversion during minimally invasive radical prostatectomy for non-metastatic prostate cancer
}

Runninghead: open conversion radical prostatectomy Stefano Luzzago ${ }^{a, b}$, Giuseppe Rosiello ${ }^{a, c}$, Angela Pecoraro ${ }^{a, d}$, Marina Deuker $^{a, e}$, Franziska Stolzenbach ${ }^{a, f}$, Francesco Alessandro Mistretta ${ }^{a, b}$, Zhe Tian $^{a}$, Gennaro Musi ${ }^{b}$, Emanuele Montanari ${ }^{\mathrm{g}}$, Shahrokh F. Shariat ${ }^{\mathrm{h}, \mathrm{il}, \mathrm{m}, \mathrm{n}}$, Fred Saad $^{\text {a }}$, Alberto Briganti ${ }^{c}$, Ottavio de Cobelli ${ }^{b, o}$, Pierre I. Karakiewicz ${ }^{a}$ ${ }^{a}$ Cancer Prognostics and Health Outcomes Unit, Division of Urology, University of Montreal Health Center, Montreal, Quebec, Canada ${ }^{b}$ Department of Urology, European Institute of Oncology, IRCCS, Milan, Italy 'Department of Urology and Division of Experimental Oncology, URI, Urological Research Institute, IRCCS San Raffaele Scientific Institute, Milan, Italy

${ }^{\mathrm{d}}$ Department of Urology, San Luigi Gonzaga Hospital, University of Turin, Turin, Italy

${ }^{\mathrm{e}}$ Department of Urology, University Hospital Frankfurt, Frankfurt am Main, Germany

fMartini Klinik, University Medical Center Hamburg-Eppendorf, Hamburg, Germany

'Department of Urology, IRCCS Fondazione Ca' Granda-Ospedale Maggiore Policlinico, University of Milan, Milan, Italy

${ }^{\mathrm{h}}$ Department of Urology, Comprehensive Cancer Center, Medical University of Vienna, Vienna, Austria

'Departments of Urology, Weill Cornell Medical College, New York, New York, USA

'Department of Urology, University of Texas Southwestern, Dallas, Texas, USA

mDepartment of Urology, Second Faculty of Medicine, Charles University, Prag, Czech Republic ${ }^{n}$ Institute for Urology and Reproductive Health, I.M. Sechenov First Moscow State Medical University, Moscow, Russia 
${ }^{\circ}$ Department of Oncology and Hemato-Oncology, University of Milan, Milan, Italy

Corresponding author:

Stefano Luzzago, MD

Department of Urology, European Institute of Oncology, IRCCS, Milan, Italy

Via Giuseppe Ripamonti, 435

20141 Milan, Italy

Tel: +3933354249298

E-mail: stefanoluzzago@gmail.com

Word count (abstract): 261

Word count (manuscript): 2467 
Abstract

Background

To test contemporary rates and predictors of open conversion at minimally invasive (laparoscopic or robotic) radical prostatectomy (MIRP).

\section{Methods}

Within the National Inpatient Sample database (2008-2015) we identified all MIRP patients and patients that underwent open conversion at MIRP. First, estimated annual percentage changes (EAPC) tested temporal trends of open conversion. Second, multivariable logistic regression models predicted open conversion at MIRP. All models were weighted and adjusted for clustering, as well as all available patient and hospital characteristics.

Results

Of 57,078 MIRP patients, 368 (0.6\%) underwent open conversion. The rates of open conversion decreased over time (from 1.80 to $0.38 \%$; EAPC:-26.0\%; $p=0.003$ ). In multivariable logistic regression models predicting open conversion, patient obesity (OR:2.10; $p<0.001)$, frailty (OR:1.45; $p=0.005)$ and Charlson Comorbidity Index $(C \mathrm{Cl}) \geq 2$ (OR:1.57; $p=0.03$ ) achieved independent predictor status.Moreover,compared to high volume hospitals, medium volume (OR:2.03; $\mathrm{p}<0.001)$ and low volume hospitals (OR:3.86; $p<0.001$ ) were associated with higher rates of open conversion.Last but not least, when the interaction between the number of patient risk factors (obesity and/or frailty and/or $\mathrm{CCl} \geq 2$ ) and hospital volume was tested, a dose-response effect was observed. Specifically, the rates of open conversion ranged from $0.3 \%$ (patients with 0 risk factors treated at high volume hospitals) to $2.2 \%$ (patients with 2-3 risk factors treated at low volume hospitals). Conclusion

Overall contemporary (2008-2015) rate of open conversion at MIRP was $0.6 \%$ and it was strongly associated with patient obesity, frailty, $\mathrm{CCl} \geq 2$ and hospital surgical volume. In consequence, these parameters should be taken into account during preoperative patients counselling, as well as in clinical and administrative decision making.

Keywords: National Inpatient Sample; radical prostatectomy; open conversion; obesity; hospital volume; frailty; Charlson Comorbidity Index 


\section{Introduction}

Over the last decades, minimally invasive radical prostatectomy (MIRP: laparoscopic or robotic RP) has become the preferred surgical approach for patients with prostate cancer $[1,2]$. Compared to open retropubic RP, MIRP offers decreased postoperative pain, shorter hospital stay and more rapid return to normal activities $[3,4]$. Moreover, MIRP was associated with comparable intermediate time oncological outcomes, relative to open retropubic RP $[5,6]$. Despite the increased use of MIRP, open conversion may occur due to intraoperative complications, failure to progress, anatomical difficulty or cancer control considerations. The rates of open conversion at MIRP have been poorly investigated [7-10]. In the era of laparoscopic surgery (2000-2005), Bhayani et al. [8] and Hu et al. [7] reported conflicting results in terms of open conversion rates at MIRP, that ranged from 1.9 to $28.6 \%$. More contemporary data were reported by Sharma et al. [10] and Weiner et al. [9]. However, Sharma et al. [10] heavily relied on patients treated in the era of robotic RP development (2004-2010). Moreover, Weiner et al. [9] focused on a short time series that spanned years 2010 to 2011. We hypothesized lower contemporary rates of open conversion at MIRP, due to increased use of the robotic technology and higher confidence of surgeons with MIRP. Moreover, we also hypothesized that several patient and hospital characteristics could predict open conversion at MIRP. To address this void, we examined a contemporary RP series (2008-2015) within a large population-based data repository and tested rates and predictors of open conversion at MIRP.

\section{Materials and methods}

Study population

We relied on the National Inpatient Sample (NIS) database (2008-2015) [11] that is composed of longitudinal hospital inpatient databases from the Healthcare Cost and Utilization Project and includes $20 \%$ of United States inpatient hospitalizations. We focused on patients aged $\geq 18$ years with primary diagnosis of non-metastatic prostate cancer (International Classification of Diseases, Ninth Revision, Clinical Modification [ICD-9CM] code 185.0), treated with RP (primary procedure ICD-9-CM code 65.0). Secondary procedure codes were used to identify laparoscopic or robotic approach (ICD-9-CM codes 54.21 and 17.42 ) and lymph node dissection (ICD-9-CM codes 40.3/40.5). 
Outcomes of interest

We focused on open conversion rates at MIRP. Patients were considered to have undergone open conversion if the codes for open conversion (ICD-9-CM codes V64.4 and V64.41) appeared during the same admission as the code for RP. This methodology was consistent with previous studies that relied on the NIS $[12,13]$. Since the majority of open conversion cases were coded as open RP + open conversion, distinction between laparoscopic and robotic MIRP was not possible.

Variables definition

Demographic characteristics consisted of: age at surgery, race (Caucasian, AfricanAmerican and Others), year of surgery, Charlson Comorbidity Index (CCI), obesity $(\mathrm{BMI} \geq 30)$, frailty, income and insurance status (private, Medicare, Medicaid and other). Specifically, $\mathrm{CCI}$ was defined according to the Deyo adaptation for ICD-9 codes-based databases [14] and categorized as $\mathrm{CCl} 0-1$ vs. $\mathrm{CCl} \geq 2$. Moreover, frailty (categorized as frail vs. non-frail patients) was defined according to the Johns Hopkins Adjusted Clinical Groups (ACG) frailty-defining diagnoses indicator and was calculated using patients' ICD-9 codes available in the NIS, as previously reported $[15,16]$.

Hospital region (Northeast, Midwest, South, West), hospital teaching vs. non-teaching status, hospital bed size (small, medium and large) and hospital annual volume (tertiles: low, medium and high), which represents the number of MIRPs performed at each participating institution during each study calendar year, were considered.

\section{Statistical analyses}

First, differences in medians and proportions were assessed by, respectively, the Kruskal-Wallis and the chi-square tests. Second, estimated annual percentage changes (EAPC) tested temporal trends of open conversion over time. Third, open conversion rates at MIRP were tested in multivariable logistic regression models. All multivariable models relied on generalized estimating equations to further adjust for clustering [11]. Subsequently, data distribution was adjusted according to the provided NIS population weights to render estimates more accurate nationally [11]. All statistical tests were twosided with a level of significance set at $p<0.05$. All analyses were performed using the $R$ software environment for statistical computing and graphics (version 3.4.1; http://www.rproject.org/). 


\section{$\underline{\text { Results }}$}

\section{Descriptive analyses and trends over time}

Of 57,078 MIRP patients, 368 (0.6\%) underwent open conversion (Table 1). Patients that underwent open conversion more frequently were obese (15.5 vs. $8.4 \% ; p<0.001)$ and frail (19.8 vs. $13.6 \% ; p<0.001)$ and more frequently had $\mathrm{CCl} \geq 2(6.2$ vs. $3.4 \% ; p=0.004)$ and low income status ( $1^{\text {st }}$ quantile: 26.9 vs. $21 \%$; $=0.02$ ). Moreover, patients that underwent open conversion less frequently received lymph node dissection ( 29.3 vs. $37.6 \%$; $p<0.001$ ). Finally, patients that underwent open conversion more frequently were treated at low volume hospitals ( 45.4 vs. $26.7 \%$; $p<0.001$ ), at non teaching hospitals ( 42.7 vs. $27 \%$; $p<0.001$ ) and at large bed size hospitals (69.3 vs. $64.2 \% ; p=0.03$ ). Time trends revealed a decrease in the rates of open conversion over time (from 1.80 to $0.38 \%$;

EAPC:-26.0\%; $p=0.003$; Figure 1).

Crude rates of open conversion according to patient and hospital characteristics

The rates of open conversion in obese vs. non obese patients were, respectively, 1.2 vs. $0.6 \%$ ( $p<0.001$; Figure $2 a)$. Additionally, the rates of open conversion in frail vs. nonfrail patients were, respectively, 1.0 vs. $0.6 \%$ ( $p<0.001$; Figure $2 b$ ). Moreover, the rates of open conversion in $\mathrm{CCl} \geq 2$ vs. $\mathrm{CCl} 0-1$ patients were, respectively, 1.2 vs. $0.6 \%$ ( $p=0.004$; Figure 2c). Finally, hospital volume and hospital teaching status resulted in differences in open conversion rates. Specifically, open conversion rates at low vs. medium vs. high volume hospitals were, respectively, $1.1 \%$ vs. $0.6 \%$ vs. $0.3 \%$ (Figure $2 \mathrm{~d}$ ). Moreover, open conversion rates at non teaching vs. teaching hospitals were, respectively, 1.0 vs. $0.5 \%$ $(p<0.001$; Figure $2 e)$. No other patient or hospital characteristics were associated with higher rates of open conversion.

Multivariable analyses predicting open conversion rates

In multivariable logistic regression models (Table 2), obesity (Odds ratio [OR]:2.10; $p<0.001)$, frailty (OR:1.45; $p=0.005)$ and $C C l \geq 2(O R: 1.57 ; p=0.03)$ were associated with higher rates of open conversion. Moreover, MIRP at medium (OR:2.03; $p<0.001)$ and low volume hospitals (OR:3.86; $\mathrm{p}<0.001$ ) was associated with higher rates of open conversion, compared to MIRP at high volume hospitals. 
Crude rates of open conversion according to obesity, frailty, $\mathrm{CCl}$ and hospital volume: doseresponse effect

The association between the number of patient risk factors (namely: obesity and/or frailty and/or $\mathrm{CCl} \geq 2$ ) and hospital volume yielded different conversion rates. At small volume hospitals, the rates of open conversion were, respectively, $0.9 \%$ vs. $1.5 \%$ vs. $2.2 \%$ in patients with 0 vs. 1 vs. 2-3 risk factors (Figure 3). At medium volume hospitals, the rates of open conversion were, respectively, $0.6 \%$ vs. $0.9 \%$ vs. $1.8 \%$ in patients with 0 vs. 1 vs. 2 3 risk factors. Finally, at high volume hospitals, the rates of open conversion were, respectively, $0.3 \%$ vs. $0.5 \%$ vs. $0.9 \%$ in patients with 0 vs. 1 vs. $2-3$ risk factors. Multivariable analyses predicting open conversion rates according to obesity, frailty, $\mathrm{CCl}$ and hospital volume: dose-response effect

The independent predictor status of the association between the number of patient risk factors (namely: obesity and/or frailty and/or $\mathrm{CCl} \geq 2$ ) and hospital volume was confirmed in multivariable logistic regression models predicting open conversion rates, which revealed a significant and gradual dose-response effect (Table 2). Specifically, compared to patients with 0 risk factors treated at high volume hospitals, the highest OR was observed in patients with 2-3 risk factors treated at low volume hospitals (OR:9.72; $\mathrm{p}<0.001)$. Conversely, the lowest OR was recorded in patients with 1 risk factor treated at high volume hospitals (OR:1.75; $p=0.01$ ).

\section{Discussion}

Only four previous studies examined the rates of open conversion at MIRP [7-10]. However, three of these reports heavily relied on patients treated in the era of laparoscopic MIRP and considered surgeons at the beginning of their learning curve $[7,8,10]$. Moreover, Weiner et al. [9] focused on a short time series that spanned years 2010 to 2011. Therefore, these data could not be representative of the current situation in the United States. Based on lack of data on contemporary rates of open conversion during MIRP, we tested temporal trends and predictors of open conversion in a large populationbased MIRP cohort $(n=57,078)$ that spanned years 2008 to 2015 . Our results showed several important findings.

First, open conversion occurred in $0.6 \%$ of contemporary MIRPs. Moreover, the rates of open conversion at MIRP decreased over time (from 1.80 to $0.38 \%$; EAPC:-26.0\%). 
Our findings are consistent with a previous temporal trend analysis (2004-2010) by Sharma et al. [10] in which the rates of open conversion at MIRP decreased from 7.2 to $0.7 \%$. However, the report of Sharma et al. [10] heavily relied on patients treated in the era of laparoscopic RP and, therefore, considered surgeons at the beginning of their robotic RP learning curve. In consequence, to the best of our knowledge, we are the first to show decreasing rates of open conversion at MIRP in the era of robotic RP (2008-2015). Several hypothetical explanations could justify this encouraging trend. First, robotic approach is increasingly being used for MIRP in the United States [1,2]. Oberlin et al. [17] reported that the performance of robotic MIRP in the United States increased from 22\% of all RP in 2003 to $85 \%$ in 2013 . Indeed, robotic MIRP was previously associated with lower rates of intraoperative and postoperative complications and with a shorter learning curve, compared to laparoscopic MIRP $[18,19]$. Second, the technique of MIRP and surgeon experience have improved over time. Unfortunately, we were unable to validate these two specific hypotheses because of inherent limitation of the NIS database. Specifically, we were unable to distinguish between open conversions of laparoscopic and robotic MIRPs, as the majority of open conversions were coded as an open procedure. Moreover, surgeon experience is unavailable within the NIS. Therefore, the observed decreasing trends of open conversion at MIRP should be tested in other large data repositories that systematically register surgical approach and surgeon experience.

Second, the rates of open conversion at MIRP varied according to patient and hospital characteristics. Specifically, obese ( 1.2 vs. $0.6 \%$; $p<0.001$ ), frail ( 1.0 vs. $0.6 \%$; $\mathrm{p}<0.001$ ) or $\mathrm{CCl} \geq 2$ (1.2 vs.0.6\%; $\mathrm{p}=0.004)$ patients experienced higher rates of open conversion, relative to their non obese, non-frail or $\mathrm{CCl}$ 0-1 counterparts. Moreover, patients treated at low and medium volume hospitals were at higher risk of open conversion, compared to patients treated at high volume hospitals ( $1.1 \%$ vs. $0.6 \%$ vs.0.3\%). These findings were confirmed in multivariable logistic regression models predicting open conversion rates, where obesity, frailty, $\mathrm{CCl} \geq 2$ and hospital volume achieved independent predictor status, even after full adjustment for all available patient and hospital characteristics. Obesity, frailty, $\mathrm{CCl} \geq 2$ and low hospital volume were previously associated with higher rates of perioperative complications in patients treated with RP [20-29]. Moreover, our findings are consistent with a previous study by Sharma et al. [10] 
that reported patient obesity and low hospital volume as independent predictors of open conversion at MIRP. However, to the best of our knowledge, the association between CCI or patient frailty and higher rates of open conversion at MIRP was never previously reported.

Third, when the interaction between the number of patient risk factors (obesity and/or frailty and/or $\mathrm{CCl} \geq 2$ ) and hospital volume was tested, a dose-response effect was observed. Specifically, the rates of open conversion ranged from $0.3 \%$ (patients with 0 risk factors treated at high volume hospitals) to $2.2 \%$ (patients with 2-3 risk factors treated at low volume hospitals). Moreover, when multivariable logistic regression models were refitted according to the number of patient risk factors and treatment at either low vs. medium vs. high volume hospitals, the independent predictor status of the dose-response effect was confirmed. Specifically, a gradual increase was recorded when a higher number of risk factors and decreasing hospital volume were combined. Compared to patients with 0 risk factors treated at high volume hospitals, the highest OR was recorded when 2-3 risk factors and low volume hospital characteristics were combined (OR: 9.72). Conversely, lower ORs were recorded when patients with 2-3 risk factors were treated at medium (OR:7.69) or high volume hospitals (OR:3.36), as well as when patients with 1 risk factor were treated at low (OR:6.51), medium (OR:3.33) and high volume hospitals (OR:1.75). Finally, lower ORs were also recorded when patients with 0 risk factors treated at low (OR:3.98) or medium volume hospitals (OR:2.04). These results are important for preoperative patient counselling, clinical decision making, as well as in health care administration.

Taken together, to the best of our knowledge, we provided the first large-scale contemporary analysis addressing open conversion rates at MIRP. Our findings showed that $0.6 \%$ of contemporary MIRPs are converted to open RP and that the rates of open conversion decreased over time. Moreover, obesity, frailty, $\mathrm{CCl} \geq 2$ and hospital volume achieved independent predictor status in multivariable models testing open conversion rates, even after adjustment for patient and hospital characteristics. Last but not least, when patients where stratified according to the number of risk factors and hospital volume, we observed a significant dose-response effect. Specifically, the rates of open conversion at MIRP ranged from $0.3 \%$ (patients with 0 risk factors treated at high volume 
hospitals) to $2.2 \%$ (patients with 2-3 risk factors treated at low volume hospitals). In consequence, it may be advisable to systematically record $\mathrm{BMI}, \mathrm{CCl}$ and frailty index in all MIRP candidates. Patients should be informed about the possibility of open conversion at MIRP, according to the number of their risk factors, as well as hospital surgical volume.

Despite its novelty, our study has limitations. First, the data are retrospective and, therefore, influenced by inherent selection bias. Second, as previously stated, we were unable to distinguish between open conversions of laparoscopic and robotic MIRPs, as the majority of open conversions were coded as an open procedure. Third, the NIS database [11] lacks of information about surgical technique or individual surgeon experience. However, Sharma et al. [10] showed that hospital and surgeon MIRP volume are two highly correlated variables $(R=0.7, p<0.001)$. We therefore believe that hospital volume could represent a good, but not perfect, surrogate of individual surgeon volume. In consequence, open conversion at MIRP should be tested in other large data repositories that systematically register surgical approach and surgeon experience. Fourth, information on performance status, ASA score, lookback period of $\mathrm{CCl}$ assessment, as well as laboratory values, are not available within the NIS database. Fifth, the NIS database does not capture specific reasons for open conversion during MIRP. Open conversion may occur due to intraoperative complications, failure to progress, anatomical difficulty, cancer control considerations or anesthesiologist's request. The impact of these specific reasons should be therefore tested in other large data repositories. Finally, since the NIS database does not provide tumor characteristics, such as stage and grade, we were unable to adjust our analyses for these variables.

\section{Conclusion}

Overall contemporary (2008-2015) rate of open conversion at MIRP was $0.6 \%$ and it was strongly associated with patient obesity, frailty, $\mathrm{CCl} \geq 2$ and hospital surgical volume. In consequence, these parameters should be taken into account during preoperative patients counselling, as well as in clinical and administrative decision making.

Acknowledgements: None

Author disclosure statement: "No competing financial interests exist." 


\section{References}

[1] Lowrance WT, Eastham JA, Savage C, Maschino AC, Laudone VP, Dechet CB, et al. Contemporary open and robotic radical prostatectomy practice patterns among urologists in the United States. J Urol 2012. doi:10.1016/j.juro.2012.01.061.

[2] Kowalczyk KJ, Levy JM, Caplan CF, Lipsitz SR, Yu HY, Gu X, et al. Temporal national trends of minimally invasive and retropubic radical prostatectomy outcomes from 2003 to 2007: Results from the 100\% medicare sample. Eur Urol 2012. doi:10.1016/j.eururo.2011.12.020.

[3] Trinh QD, Sammon J, Sun M, Ravi P, Ghani KR, Bianchi M, et al. Perioperative outcomes of robot-assisted radical prostatectomy compared with open radical prostatectomy: Results from the nationwide inpatient sample. Eur Urol 2012. doi:10.1016/j.eururo.2011.12.027.

[4] Nelson B, Kaufman M, Broughton G, Cookson MS, Chang SS, Herrell SD, et al. Comparison of Length of Hospital Stay Between Radical Retropubic Prostatectomy and Robotic Assisted Laparoscopic Prostatectomy. J Urol 2007. doi:10.1016/j.juro.2006.10.070.

[5] Hu JC, Gandaglia G, Karakiewicz PI, Nguyen PL, Trinh QD, Shih YCT, et al. Comparative effectiveness of robot-assisted versus open radical prostatectomy cancer control. Eur Urol 2014. doi:10.1016/j.eururo.2014.02.015.

[6] Hu JC, O’Malley P, Chughtai B, Isaacs A, Mao J, Wright JD, et al. Comparative Effectiveness of Cancer Control and Survival after Robot-Assisted versus Open Radical Prostatectomy. J Urol 2017. doi:10.1016/j.juro.2016.09.115.

[7] Hu JC, Hevelone ND, Ferreira MD, Lipsitz SR, Choueiri TK, Sanda MG, et al. Patterns of Care for Radical Prostatectomy in the United States From 2003 to 2005. J Urol 2008;180:1969-74. doi:10.1016/j.juro.2008.07.054.

[8] Bhayani SB, Pavlovich CP, Strup SE, Dahl DM, Landman J, Fabrizio MD, et al. Laparoscopic radical prostatectomy: A multi-institutional study of conversion to open surgery. Urology 2004;63:99-102. doi:10.1016/j.urology.2003.08.018. 
[9] Weiner AB, Murthy P, Richards KA, Patel SG, Eggener SE. Population based analysis of incidence and predictors of open conversion during minimally invasive radical prostatectomy. J Urol 2015;193:826-31. doi:10.1016/j.juro.2014.09.113.

[10] Sharma V, Meeks JJ. Open conversion during minimally invasive radical prostatectomy: Impact on perioperative complications and predictors from national data. J Urol 2014. doi:10.1016/j.juro.2014.06.029.

[11] Healthcare Cost and Utilization Project (HCUP). HCUP NIS Database Documentation. Agency Healthc Res Qual Rockville, MD 2018.

[12] Moghadamyeghaneh Z, Masoomi H, Mills SD, Carmichael JC, Pigazzi A, Nguyen NT, et al. Outcomes of conversion of laparoscopic colorectal surgery to open surgery. J Soc Laparoendosc Surg 2015;18. doi:10.4293/JSLS.2014.00230.

[13] Samreen S, Fluck M, Hunsinger M, Wild J, Shabahang M, Blansfield JA. Laparoscopic versus robotic adrenalectomy: a review of the national inpatient sample. J Robot Surg 2019;13:69-75. doi:10.1007/s11701-018-0808-3.

[14] Deyo RA, Cherkin DC, Ciol MA. Adapting a clinical comorbidity index for use with ICD-9-CM administrative databases. J Clin Epidemiol 1992. doi:10.1016/08954356(92)90133-8.

[15] Mclsaac DI, Bryson GL, Van Walraven C. Association of frailty and 1-year postoperative mortality following major elective noncardiac surgery: A populationbased cohort study. JAMA Surg 2016. doi:10.1001/jamasurg.2015.5085.

[16] Nieman CL, Pitman KT, Tufaro AP, Eisele DW, Frick KD, Gourin CG. The effect of frailty on short-term outcomes after head and neck cancer surgery. Laryngoscope 2018. doi:10.1002/lary.26735.

[17] Oberlin DT, Flum AS, Lai JD, Meeks JJ. The effect of minimally invasive prostatectomy on practice patterns of American urologists. Urol Oncol Semin Orig Investig 2016. doi:10.1016/j.urolonc.2016.01.008. 
[18] Ahlering TE. Robotic vesus laparoscopic radical prostatectomy. Nat Clin Pract Urol 2004;1:58-9. doi:10.1038/ncpuro0040.

[19] Hu JC, Nelson RA, Wilson TG, Kawachi MH, Ramin SA, Lau C, et al. Perioperative complications of laparoscopic and robotic assisted laparoscopic radical prostatectomy. J Urol 2006. doi:10.1016/S0022-5347(05)00156-4.

[20] Knipper S, Mazzone E, Mistretta FA, Palumbo C, Tian Z, Briganti A, et al. Impact of obesity on perioperative outcomes at robotic-assisted and open radical prostatectomy: results from the National Inpatient Sample. Urology 2019. doi:10.1016/j.urology.2019.05.053.

[21] Lascano D, Pak JS, Kates M, Finkelstein JB, Silva M, Hagen E, et al. Validation of a frailty index in patients undergoing curative surgery for urologic malignancy and comparison with other risk stratification tools. Urol Oncol Semin Orig Investig 2015. doi:10.1016/j.urolonc.2015.06.002.

[22] Suskind AM, Jin C, Cooperberg MR, Finlayson E, Boscardin WJ, Sen S, et al. Preoperative Frailty Is Associated With Discharge to Skilled or Assisted Living Facilities After Urologic Procedures of Varying Complexity. Urology 2016. doi:10.1016/j.urology.2016.03.073.

[23] Johnson SC, Packiam VT, Golan S, Cohen AJ, Nottingham CU, Smith ND. The Effect of Obesity on Perioperative Outcomes for Open and Minimally Invasive Prostatectomy. Urology 2017;100:111-6. doi:10.1016/j.urology.2016.11.028.

[24] Abufaraj M, Mari A, Mansy K, Sievert KD. Obesity and its implications on oncological urological surgery. Curr Opin Urol 2017;27:446-55. doi:10.1097/MOU.0000000000000429.

[25] Campeggi A, Xylinas E, Ploussard G, Ouzaid I, Fabre A, Allory Y, et al. Impact of body mass index on perioperative morbidity, oncological, and functional outcomes after extraperitoneal laparoscopic radical prostatectomy. Urology 2012;80:576-84. doi:10.1016/j.urology.2012.04.066. 
[26] Castle EP, Atug F, Woods M, Thomas R, Davis R. Impact of body mass index on outcomes after robot assisted radical prostatectomy. World J Urol 2008;26:91-5. doi:10.1007/s00345-007-0217-0.

[27] Peard L, Goodwin J, Hensley P, Dugan A, Bylund J, Harris AM. Examining and Understanding Value: The Impact of Preoperative Characteristics, Intraoperative Variables, and Postoperative Complications on Cost of Robot-Assisted Laparoscopic Radical Prostatectomy. J Endourol 2019;33:541-8. doi:10.1089/end.2019.0066.

[28] Froehner M, Garbrecht B, Hakenberg OW, Koch R, Litz RJ, Oehlschlaeger S, et al. Changing comorbidity classification patterns at radical prostatectomy during a 10year period. Urol Oncol Semin Orig Investig 2007;25:26-31. doi:10.1016/j.urolonc.2006.02.004.

[29] Trinh QD, Bjartell A, Freedland SJ, Hollenbeck BK, Hu JC, Shariat SF, et al. A systematic review of the volume-outcome relationship for radical prostatectomy. Eur Urol 2013. doi:10.1016/j.eururo.2013.04.012. 
List of abbreviations

RP: radical prostatectomy

MIRP: minimally invasive radical prostatectomy

NIS: National Inpatient Sample

ICD-9-CM: International Classification of Diseases, Ninth Revision, Clinical Modification

$\mathrm{CCl}$ : Charlson Comorbidity Index

ACG: Johns Hopkins Adjusted Clinical Groups

EAPC: estimated annual percentage changes

BMI: Body Mass Index 


\section{Figure Legends}

Open conversion rates minimally invasive radical prostatectomy

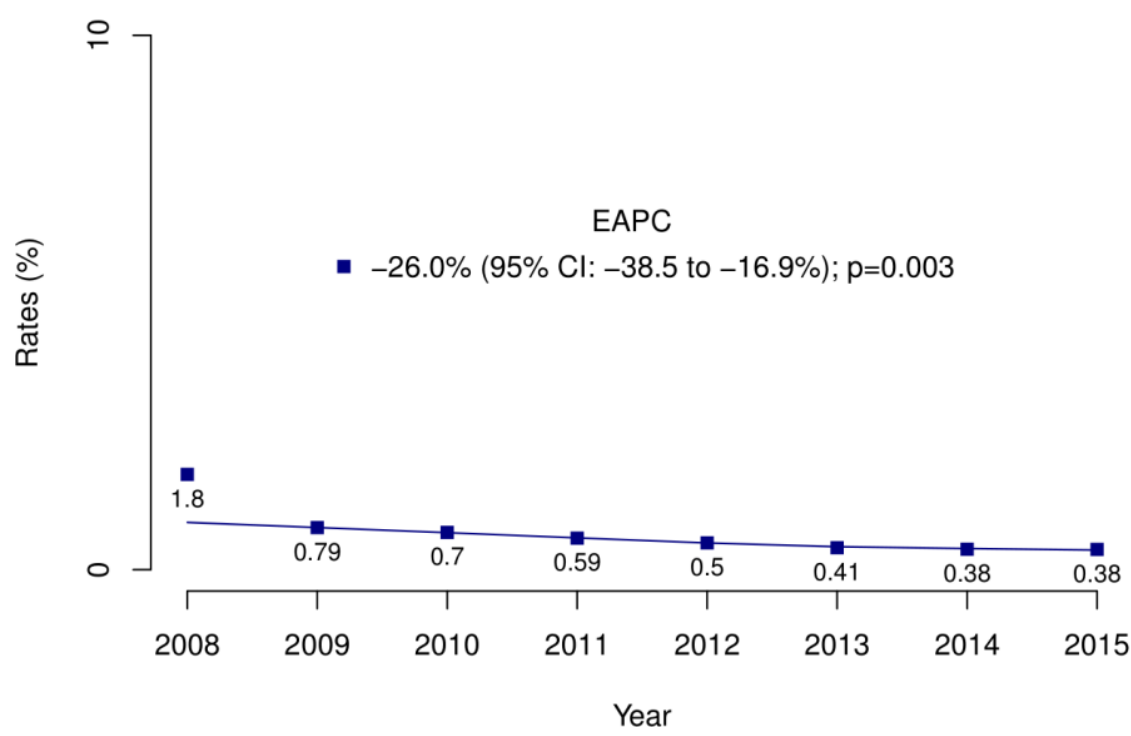

Figure 1. Annual rates of open conversion in patients treated with minimally invasive prostatectomy identified within the National (Nationwide) Inpatient Sample (NIS) database from 2008 to 2015 . EAPC: estimated annual percentage changes 
A B

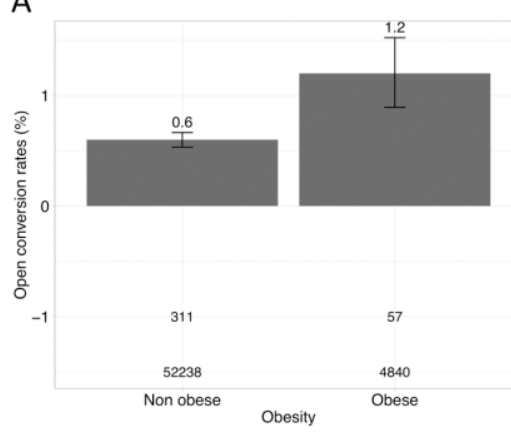

B

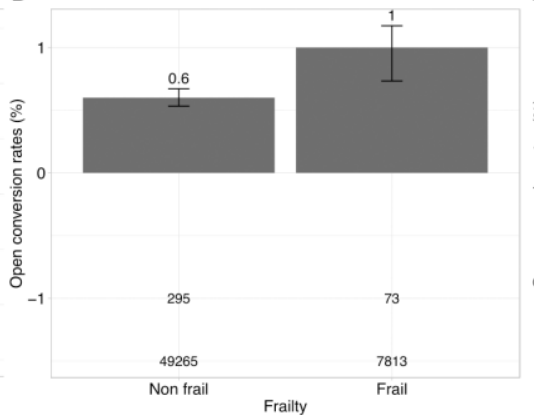

C

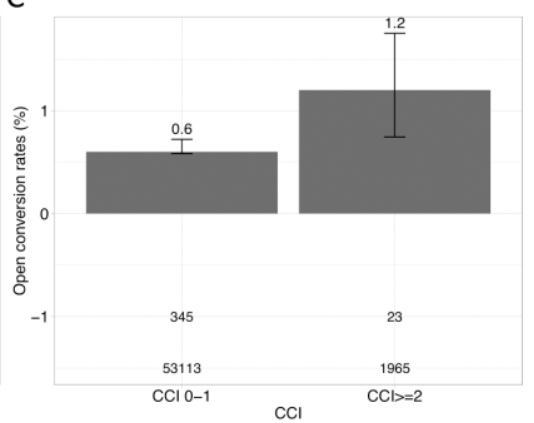

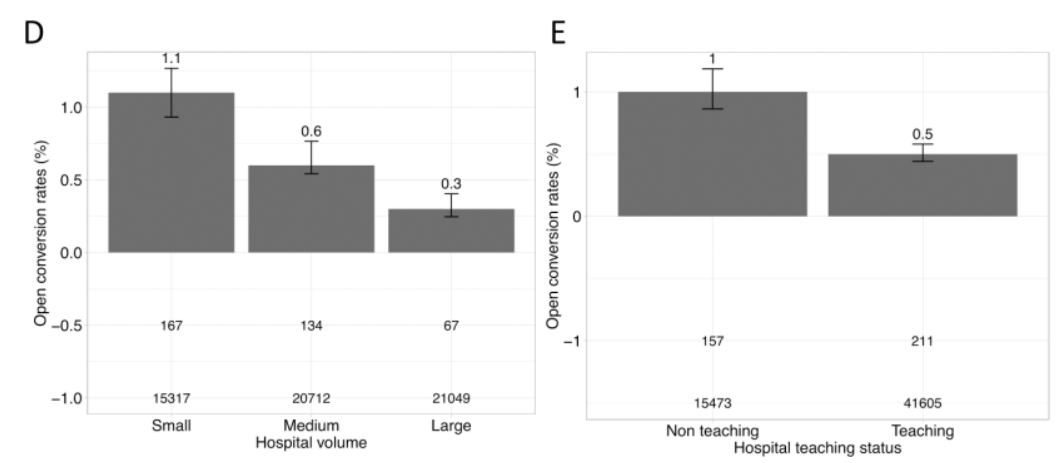

Figure 2. Barplots depicting crude rates of open conversion in patients treated with minimally invasive radical prostatectomy (unweighted population) identified within National (Nationwide) Inpatient Sample (NIS) database from 2008 to 2015. A) Obesity; B) Frailty; C) Charlson Comorbidity Index (CCI); D) Hospital volume; Hospital teaching status 


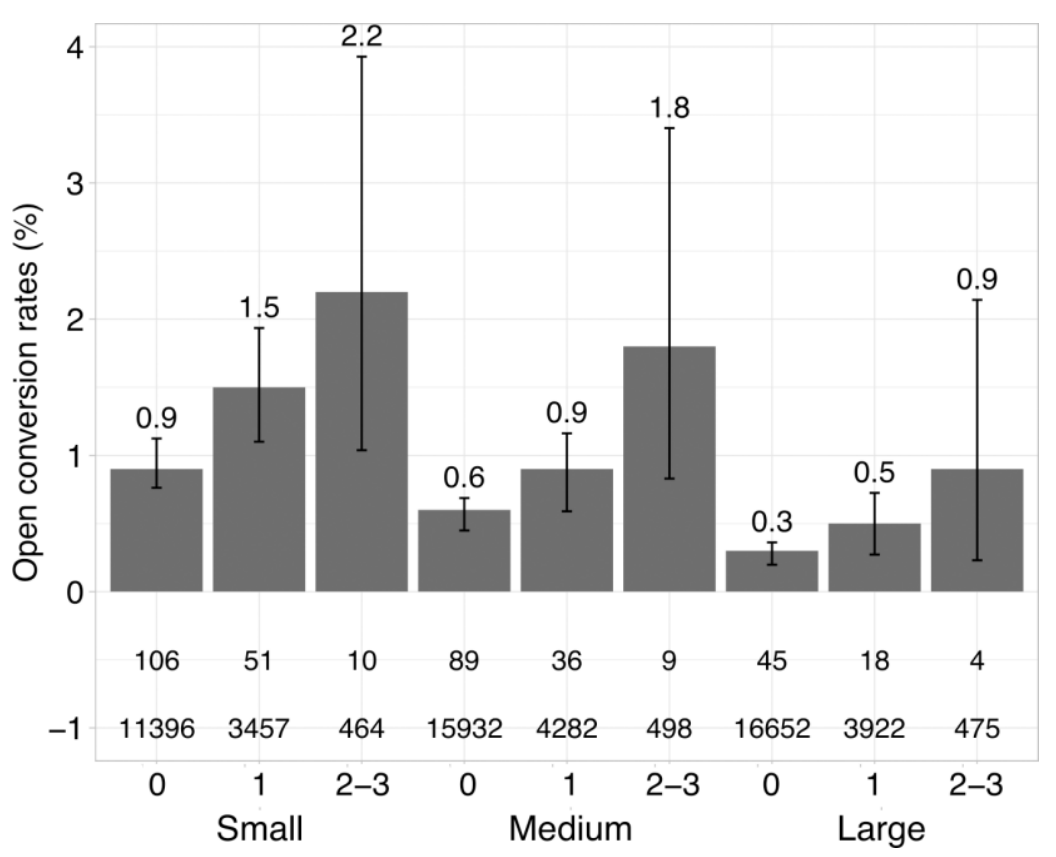

Figure 3. Barplots depicting crude rates of open conversion in patients treated with minimally invasive radical prostatectomy (unweighted population) identified within National (Nationwide) Inpatient Sample (NIS) database from 2008 to 2015. Patients are stratified according to hospital volume and according to the number of risk factors (obesity, frailty, Charlson Comorbidity Index) 
Table 1. Descriptive characteristics of $\mathbf{5 7} 078$ minimally invasive radical prostatectomy patients (unweighted population) identified within National (Nationwide) Inpatient Sample (NIS) database from 2008 to 2015 and stratified according to presence or absence of open conversion

\begin{tabular}{|c|c|c|c|c|}
\hline - & & $\begin{array}{c}\text { Open conversion: } \\
\text { Yes } \\
(n=368 ; 0.6 \%)\end{array}$ & $\begin{array}{c}\text { Open conversion: } \\
\text { No } \\
(n=56710 ; 99.4 \%)\end{array}$ & $\begin{array}{c}\mathrm{p}- \\
\text { value }\end{array}$ \\
\hline Age (years) & Median (IQR) & $63(57-67)$ & $62(57-67)$ & 0.09 \\
\hline \multirow[t]{3}{*}{ Race, n (\%) } & Caucasian & $241(65.5)$ & 39054 (68.9) & 0.07 \\
\hline & $\begin{array}{l}\text { African- } \\
\text { American }\end{array}$ & $53(14.4)$ & $6091(10.7)$ & \\
\hline & Other & $74(20.1)$ & $11565(20.4)$ & \\
\hline \multirow[t]{2}{*}{$\mathrm{CCl}, \mathrm{n}(\%)$} & $0-1$ & $345(93.8)$ & $54768(96.6)$ & 0.004 \\
\hline & $\geq 2$ & $23(6.2)$ & $1942(3.4)$ & \\
\hline \multirow[t]{2}{*}{ Obesity, n (\%) } & Obese & $311(84.5)$ & $51927(91.6)$ & $<0.001$ \\
\hline & Non obese & $57(15.5)$ & $4783(8.4)$ & \\
\hline \multirow[t]{2}{*}{ Frailty, n (\%) } & Non-frail & $295(80.2)$ & $48970(86.4)$ & $<0.001$ \\
\hline & Frail & $73(19.8)$ & $7740(13.6)$ & \\
\hline \multirow{2}{*}{$\begin{array}{c}\text { Year of diagnosis, } \mathrm{n} \\
(\%)\end{array}$} & $2008-2011$ & $257(69.8)$ & 30355 (53.5) & $<0.001$ \\
\hline & 2012-2015 & $111(30.2)$ & 26355 (46.5) & \\
\hline \multirow{2}{*}{$\begin{array}{c}\text { Lymph node } \\
\text { dissection, } \mathrm{n}(\%)\end{array}$} & No & $260(70.7)$ & $35387(62.4)$ & 0.001 \\
\hline & Yes & $108(29.3)$ & $21323(37.6)$ & \\
\hline \multirow[t]{4}{*}{ Insurance status, n (\%) } & Medicare & $126(34.2)$ & $18428(32.5)$ & 0.09 \\
\hline & Medicaid & $14(3.8)$ & $1174(2.1)$ & \\
\hline & Private & $213(57.9)$ & $34822(61.4)$ & \\
\hline & Other & $15(4.1)$ & $2286(4)$ & \\
\hline \multirow[t]{2}{*}{ Region, n (\%) } & Midwest & $100(27.2)$ & $14531(25.6)$ & 0.02 \\
\hline & Northeast & $50(13.6)$ & $10808(19.1)$ & \\
\hline
\end{tabular}




\begin{tabular}{|c|c|c|c|c|}
\hline & South & $135(36.7)$ & $21017(37.1)$ & \\
\hline & West & $83(22.6)$ & $10354(18.3)$ & \\
\hline Income, $\mathrm{n}(\%)$ & $1^{\text {st }}$ quantile & 99 (26.9) & $11894(21)$ & 0.02 \\
\hline & $2^{\text {nd }}$ quantile & $87(23.6)$ & $12907(22.8)$ & \\
\hline & $3^{\text {rd }}$ quantile & $88(23.9)$ & $14775(26.1)$ & \\
\hline & $4^{\text {th }}$ quantile & $94(25.5)$ & $17134(30.2)$ & \\
\hline $\begin{array}{l}\text { Annual hospital } \\
\text { volume, n (\%) }\end{array}$ & Low & $167(45.4)$ & $15150(26.7)$ & $<0.001$ \\
\hline & Medium & $134(36.4)$ & $20578(36.3)$ & \\
\hline & High & $67(18.2)$ & $20982(37)$ & \\
\hline Teaching status, $\mathbf{n}(\%)$ & Teaching & $211(57.3)$ & $41394(73)$ & $<0.001$ \\
\hline & Non teaching & $157(42.7)$ & $15316(27)$ & \\
\hline Hospital bedsize, n (\%) & Small & $33(9)$ & 7638 (13.5) & 0.03 \\
\hline & Medium & $80(21.7)$ & $12674(22.3)$ & \\
\hline & Large & $255(69.3)$ & $36398(64.2)$ & \\
\hline
\end{tabular}

IQR: interquartile range 
Table 2. Multivariable logistic regression models predicting open conversion rates in patients treated with minimally invasive radical prostatectomy within the National Inpatient Sample database (2008-2015). All models were weighted and adjusted for clustering. Abbreviations: $\mathrm{CCl}=$ Charlson Comorbidity Index, $\mathrm{OR}=$ odds ratio, $\mathrm{Cl}=$ confidence interval

\begin{tabular}{|c|c|c|c|c|c|}
\hline & & \multicolumn{2}{|c|}{ Model 1} & \multicolumn{2}{|c|}{ Model 2} \\
\hline & & OR (95\% Cl) & $\begin{array}{c}\text { p- } \\
\text { value }\end{array}$ & OR $(95 \% \mathrm{Cl})$ & $\begin{array}{c}p- \\
\text { value }\end{array}$ \\
\hline Obesity & Yes vs. No & $\begin{array}{c}2.10(1.57- \\
2.83)\end{array}$ & $<0.001$ & - & - \\
\hline Frailty & Frail vs. Non frail & $\begin{array}{c}1.45(1.11- \\
1.88)\end{array}$ & 0.005 & - & - \\
\hline $\mathrm{CCl}$ & $\geq 2$ vs. $0-1$ & $\begin{array}{c}1.57(1.05- \\
2.35)\end{array}$ & 0.03 & - & - \\
\hline \multirow[t]{2}{*}{$\begin{array}{l}\text { Hospital } \\
\text { volume }\end{array}$} & Medium vs. High & $\begin{array}{c}2.03(1.37- \\
3.00)\end{array}$ & $<0.001$ & - & - \\
\hline & Low vs. High & $\begin{array}{c}.86(2.65- \\
5.63)\end{array}$ & $<0.001$ & - & - \\
\hline $\begin{array}{l}\text { Hospital } \\
\text { volume + } \\
\text { number of } \\
\text { patient risk } \\
\text { factors (Obesity } \\
\text { and/or Frailty } \\
\text { and/or } \mathrm{CCl} \geq 2 \text { ) }\end{array}$ & High volume +0 risk factors & - & - & Ref. & - \\
\hline
\end{tabular}




\begin{tabular}{|c|c|c|c|c|c|}
\hline & High volume +1 risk factor & - & - & $\begin{array}{c}1.75(1.12- \\
2.73)\end{array}$ & 0.01 \\
\hline & High volume $+2-3$ risk factors & - & - & $\begin{array}{c}3.36(1.39- \\
8.17)\end{array}$ & 0.007 \\
\hline & $\begin{array}{c}\text { Medium volume }+0 \text { risk } \\
\text { factors }\end{array}$ & - & - & $\begin{array}{c}2.04(1.34- \\
3.12)\end{array}$ & $<0.001$ \\
\hline & $\begin{array}{c}\text { Medium volume }+1 \text { risk } \\
\text { factor }\end{array}$ & - & - & $\begin{array}{c}3.33(2.00- \\
5.55)\end{array}$ & $<0.001$ \\
\hline & $\begin{array}{c}\text { Medium volume }+2-3 \text { risk } \\
\text { factors }\end{array}$ & - & - & $\begin{array}{c}7.69(3.07- \\
19.27)\end{array}$ & $<0.001$ \\
\hline & Low volume +0 risk factors & - & - & $\begin{array}{c}3.98(2.63- \\
6.02)\end{array}$ & $<0.001$ \\
\hline & Low volume +1 risk factor & - & - & $\begin{array}{c}6.51(4.07- \\
10.41)\end{array}$ & $<0.001$ \\
\hline & Low volume $+2-3$ risk factors & - & - & $\begin{array}{c}9.72(4.78- \\
19.76)\end{array}$ & $<0.001$ \\
\hline $\begin{array}{l}\text { Age categories } \\
\text { (years) }\end{array}$ & $66-74$ vs. $\leq 65$ & $\begin{array}{c}1.07(0.80- \\
1.42)\end{array}$ & 0.6 & $\begin{array}{c}1.05(0.79- \\
1.40)\end{array}$ & 0.7 \\
\hline & $\geq 75$ vs. $\leq 65$ & $\begin{array}{c}1.38(0.70- \\
2.71)\end{array}$ & 0.3 & $\begin{array}{c}1.35(0.69- \\
2.64)\end{array}$ & 0.4 \\
\hline $\begin{array}{l}\text { Year of } \\
\text { diagnosis }\end{array}$ & & $\begin{array}{c}0.74(0.69- \\
0.79)\end{array}$ & $<0.001$ & $\begin{array}{c}0.74(0.69- \\
0.79)\end{array}$ & $<0.001$ \\
\hline Race & $\begin{array}{c}\text { African-American vs. } \\
\text { Caucasian }\end{array}$ & $\begin{array}{c}1.32(0.97- \\
1.77)\end{array}$ & 0.08 & $\begin{array}{c}1.32(0.97- \\
1.78)\end{array}$ & 0.07 \\
\hline
\end{tabular}




\begin{tabular}{|c|c|c|c|c|c|}
\hline & Other vs. Caucasian & $\begin{array}{c}0.99(0.75- \\
1.30)\end{array}$ & 0.9 & $\begin{array}{c}0.99(0.75- \\
1.30)\end{array}$ & 0.9 \\
\hline $\begin{array}{l}\text { Lymph node } \\
\text { dissection }\end{array}$ & Yes vs. No & $\begin{array}{c}0.91(0.72- \\
1.14)\end{array}$ & 0.4 & $\begin{array}{c}0.91(0.72- \\
1.14)\end{array}$ & 0.4 \\
\hline Teaching status & Non teaching vs. Teaching & $\begin{array}{c}1.21(0.92- \\
1.58)\end{array}$ & 0.2 & $\begin{array}{c}1.21(0.92- \\
1.58)\end{array}$ & 0.2 \\
\hline \multirow[t]{3}{*}{ Region } & Northeast vs. Midwest & $\begin{array}{c}0.83(0.55- \\
1.25)\end{array}$ & 0.4 & $\begin{array}{c}0.83(0.55- \\
1.24)\end{array}$ & 0.3 \\
\hline & South vs. Midwest & $\begin{array}{c}1.01(0.74- \\
1.39)\end{array}$ & 0.9 & $\begin{array}{c}1.01(0.74- \\
1.39)\end{array}$ & 0.9 \\
\hline & West vs. Midwest & $\begin{array}{c}1.20(0.83- \\
1.76)\end{array}$ & 0.3 & $\begin{array}{c}1.19(0.82- \\
1.75)\end{array}$ & 0.3 \\
\hline \multirow[t]{3}{*}{ Insurance } & Medicaid vs. Medicare & $\begin{array}{c}1.69(0.90- \\
3.15)\end{array}$ & 0.1 & $\begin{array}{c}1.70(0.91- \\
3.17)\end{array}$ & 0.1 \\
\hline & Private vs. Medicare & $\begin{array}{c}0.99(0.75- \\
1.32)\end{array}$ & 0.9 & $\begin{array}{c}0.99(0.75- \\
1.32)\end{array}$ & 0.9 \\
\hline & Other vs. Medicare & $\begin{array}{c}0.88(0.46- \\
1.69)\end{array}$ & 0.7 & $\begin{array}{c}0.88(0.46- \\
1.69)\end{array}$ & 0.7 \\
\hline \multirow[t]{2}{*}{ Income } & 2nd vs. 1st quantile & $\begin{array}{c}0.79(0.59- \\
1.07)\end{array}$ & 0.2 & $\begin{array}{c}0.79(0.59- \\
1.07)\end{array}$ & 0.2 \\
\hline & 3rd vs. 1st quantile & $\begin{array}{c}0.73(0.55- \\
1.03)\end{array}$ & 0.1 & $\begin{array}{c}0.73(0.55- \\
1.03)\end{array}$ & 0.1 \\
\hline & 4th vs. 1 st quantile & $\begin{array}{c}0.72(0.53- \\
1.01)\end{array}$ & 0.05 & $\begin{array}{c}0.72(0.53- \\
1.01)\end{array}$ & 0.05 \\
\hline
\end{tabular}


Page $\mathbf{2 4}$ of $\mathbf{2 4}$

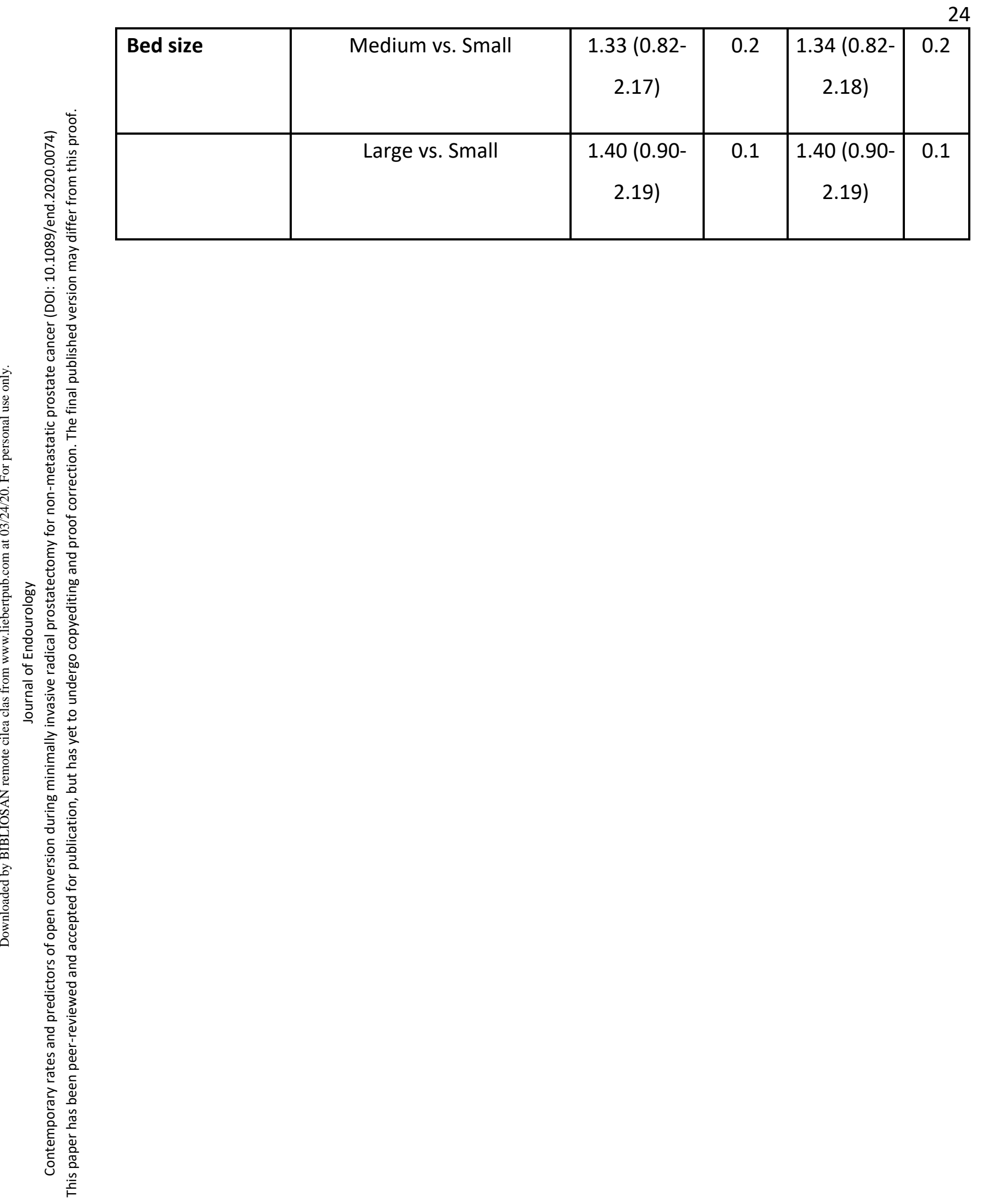

